\title{
Brève note complémentaire sur l'aire de répartition de Tetrapetalonema streptocerca en Afrique de l'ouest
}

\author{
O. BA et A. PROST*
}

\begin{abstract}
RÉSUMÉ. Un seul cas de $T$. streptocerca a été mis en évidence par l'examen de 783 personnes au sud de la Côte-d'Ivoire, tandis que l'absence du parasite chez 1559 habitants de la région des plateaux togolais conduit à la conclusion que la transmission locale de cette filaire ne dépasse pas au Togo la latitude $6^{\circ}$ Nord.
\end{abstract}

\section{Complementary Note about the repartition of Tetrapetalonema strepto- cerca in West Africa.}

SUMMARY. A single case of $T$. streptocerca was identified through the examination of 783 people in Southern Ivory-Coast, whereas the absence of the parasite among 1.559 people living in the plateaux area in Togo suggests that local transmission of the filaria does not occur in that country north of the $6^{\circ}$.

C'est dans cette optique que nous avons examiné en 1979-1980 un total de 1559 individus habitant 8 villages de la région des plateaux togolais (entre $6^{\circ} 30^{\prime}$ et $8^{\circ}$ de latitude nord) et 783 habitants de 4 villages forestiers ivoiriens, tous autochtones, les migrants étant exclus de l'échantillon.

Une biopsie cutanée exsangue a été prélevée à chaque individu, sans distinction d'âge, au niveau d'une des omoplates, site électif de la microfiladermie (Kershaw et al. 1954), en utilisant une pince à sclérectomie. Les fragments de peau ont été incubés durant 24 heures dans les puits d'un plateau de microtitration contenant $0,3 \mathrm{ml}$. de sérum physiologique. Celui-ci a été ensuite filtré sur des filtres Millipore (R) de 0,5 microns, séchés à l'air, puis colorés à l'hémalun de Mayer à chaud avant d'être examinés au microscope.

\footnotetext{
* Programme de Lutte contre l'Onchocercose B.P. 549, Ouagadougou, Haute-Volta.
}

En 1978, II9 cas d'infection streptocerquienne avaient été dépistés parmi 3500 habitants de la Volta Region au Ghana (Prost 1979). Ces résultats, acquis dans une zone frontalière du Togo, rendaient probable la présence de $T$. streptocerca dans cet État bien qu'elle n'y ait jamais été signalée. De même était-il probable que la zone invoirienne fût propice à la transmission de la filaire.

Accepté le 24 août 1982 . 
Les villages examinés ont été les suivants :

\begin{tabular}{ccc}
\hline Villages & $\begin{array}{c}\text { Coordonnées } \\
\text { géographiques }\end{array}$ & $\begin{array}{c}\text { Nombre } \\
\text { d'examinés }\end{array}$ \\
\hline Togo & & \\
Dayes-Dodji & $7^{\circ} 37^{\prime} \mathrm{N}-0^{\circ} 37^{\prime} \mathrm{E}$ & 411 \\
Konigbo & $7^{\circ} 37^{\prime} \mathrm{N}-1^{\circ} 04^{\prime} \mathrm{E}$ & 152 \\
Amoutta & $7^{\circ} 20^{\prime} \mathrm{N}-0^{\circ} 43^{\prime} \mathrm{E}$ & 104 \\
Kouma-Kunda & $6^{\circ} 58^{\prime} \mathrm{N}-0^{\circ} 35^{\prime} \mathrm{E}$ & 221 \\
Tokpo & $6^{\circ} 46^{\prime} \mathrm{N}-0^{\circ} 55^{\prime} \mathrm{E}$ & 125 \\
Adedakope & $7^{\circ} 20^{\prime} \mathrm{N}-1^{\circ} 0^{\prime} \mathrm{E}$ & 202 \\
Gebakui & $7^{\circ} 05^{\prime} \mathrm{N}-0^{\circ} 45^{\prime} \mathrm{E}$ & 227 \\
Abossomkope & $8^{\circ} 06^{\prime} \mathrm{N}-0^{\circ} 37^{\prime} \mathrm{E}$ & 117 \\
Côte d'Ivoire & & \\
Wa & & 145 \\
Gbentopleu & $7^{\circ} 27^{\prime} \mathrm{N}-8^{\circ} 10^{\prime} \mathrm{E}$ & 185 \\
Nimpleu & $7^{\circ} 27^{\prime} \mathrm{N}-8^{\circ} 22^{\prime} \mathrm{E}$ & 248 \\
Blekoum & $6^{\circ} 22^{\prime} \mathrm{N}-8^{\circ} 06^{\circ} \mathrm{E}$ & 205 \\
\hline
\end{tabular}

Sur ces 2342 examens, $T$. streptocerca n'a été identifiée qu'une seule fois, chez une jeune femme âgée de 25 ans et habitant le village de Nimpleu, dans la région de Danané en Côte-d'Ivoire, à la frontière de la Guinée et du Libéria. L'interrogatoire a confirmé qu'elle était née au village et ne l'avait jamais quitté, si ce n'est lors de brefs déplacements dans la région.

Sa charge parasitaire était faible : 3 microfilaires. Ce cas confirme la rareté de cette filariose en Côte-d'Ivoire où trois cas seulement ont été signalés jusqu'ici (Pfister 1954).

L'absence de $T$. streptocerca au Togo est plus surprenante car la région étudiée est contiguë de celle dans laquelle ont été reconnus des foyers de transmission locale au Ghana (Prost 1979) ; elle présente les mêmes caractéristiques écologiques, avec des forêts mésophiles, un relief fortement accidenté, et des altitudes comparables. La seule différence méthodologique pourrait résider dans le fait que les biopsies ont été pratiquées au niveau de l'omoplate, et non de la crête iliaque comme au Ghana. Mais ceci impliquerait que le site électif des microfilaires ne soit pas l'épaule en Afrique de l'ouest, contrairement aux observations de Kershaw et al. (1954) au Cameroun.

\section{BIBLIOGRAPHIE}

Kershaw W. E., Duke B. O. L., Budden F. H. : The distribution of the microfilariae of Acanthocheilonema streptocerca in the skin of man. Trans. Roy. Soc. Trop. Med. Hyg., 1954, 48, 287 .

PFISTER R. : Résultats d'une enquête sur les porteurs de microfilaires en Afrique occidentale française. Bull. Soc. Pathol. Exot., 1954, 47, 408-4I I.

Prost A. : Éléments complémentaires sur la répartition et la fréquence de Tetrapetalonema (Esslingerial streptocerca (Macfie et Corson, 1922) en Afrique de l'ouest. Ann. Parasitol. Hum. Comp., 1979, 54, 93-97. 